\title{
Unscented iSAM: A Consistent Incremental Solution to Cooperative Localization and Target Tracking
}

\author{
Guoquan Huang, Robert Truax, Michael Kaess, and John J. Leonard
}

\begin{abstract}
In this paper, we study the problem of cooperative localization and target tracking (CLATT), i.e., a team of mobile robots use their onboard sensors' measurements to cooperatively track multiple moving targets, and propose a novel unscented incremental smoothing and mapping (U-iSAM) approach. The proposed method attains reduced linearization errors by using the unscented transformation and correct observability properties by imposing observability constraints on the unscented transformation when computing measurement Jacobians. In particular, we, for the first time ever, analyze the observability properties of the batch maximum a posteriori (MAP)-based CLATT system, and show that in the case of no prior, the Hessian (information) matrix has a nullspace of dimension three. However, this may not be the case when the Jacobians (and thus the Hessian) are computed numerically through the unscented transformation. To ensure that the $U$ iSAM possesses correct observability (i.e., the nullspace of its Hessian is of dimension three), we project the measurement Jacobians computed by the standard unscented transformation onto the observable subspace. The proposed algorithm is validated through extensive Monte-Carlo simulations.
\end{abstract}

\section{INTRODUCTION}

When a team of mobile robots working in dynamic environments, it is necessary for the robots to simultaneously determine their poses (positions and orientations) and the kinematic states of moving objects (targets), such as positions and velocities. This is due to the fact that jointly estimating the robots and targets results in better accuracy for the robots' position estimates, as compared to localizing the robots by ignoring the targets [1]. This is the problem of cooperative localization and target tracking (CLATT), a typical nonlinear estimation problem arising in many practical applications such as surveillance [2]. In this paper, we study in-depth this CLATT problem and introduce a novel consistent incremental estimation algorithm.

Although many different algorithms are available for solving the CLATT problem, among them the extended Kalman filter (EKF) remains a popular choice primarily due to its relatively low processing requirements and its ease of implementation [1]. However, its performance depends on the magnitude of the linearization errors. In order to reduce the linearization errors, the unscented transformation (statistical linearization) used by the unscented Kalman filter (UKF) [3] is appealing, which deterministically samples the nonlinear model around the current state estimate and employs linear regression to improve the accuracy of the linear

This work was partially supported by ONR grants N00014-12-1-0093, N00014-10-1-0936, N00014-11-1-0688, and N00014-12-10020. The authors are with the Computer Science and Artificial Intelligence Laboratory, Massachusetts Institute of Technology, Cambridge, MA 02139, USA. Email: \{gqhuang|robtruax|kaess|jleonard\}@mit.edu approximation [4]. Nevertheless, any (explicit or implicit) linearization-based filtering approach, marginalizes all but the current state and hence is unable to correct linearization errors involving previous states.

For this reason, smoothing approaches, either in batch or incremental fashion, have become prevailing in robotics (see [5]-[10] and references therein). In particular, a batch maximum a posteriori (MAP) estimator [11] computes the estimates for the states at all time steps using all available measurements. This allows continuous relinearization around all the states, which can greatly reduce the linearization errors. However, since the size of the state vector in the batch-MAP estimator increases continuously over time, the processing and memory requirements become too high for real-time operation in large-scale problems (e.g., when a team of robots work for an extended period of time). To reduce the computational complexity of the batch approach, the incremental smoothing and mapping (iSAM) algorithm [7] reduces computation by employing factorization-updating methods which allow reusing the information-matrix factorization available from previous time steps. Computationally demanding procedures, such as relinearization and batch factorization, are only performed intermittently. While the iSAM was shown to perform well in robotic mapping applications [7], it still suffers from large linearization errors in dynamic environments, which degrade its performance, for example, in the CLATT problem under consideration.

In order to mitigate the negative impact due to linearization errors, in this paper we introduce an unscented-iSAM (UiSAM) algorithm for the CLATT problem. In particular, the proposed U-iSAM computes the Jacobians, and thus the Hessian, by applying the unscented transformation, which has shown to greatly reduce linearization errors [3]. However, by performing observability analysis, we find that the Hessian (information) matrix computed numerically through the unscented transformation may have a nullspace that has different dimensions from that of the optimal (up to linearization errors) batch-MAP estimator. This implies that spurious (nonexistent) information is gained from the measurements, which can lead to estimation inconsistency (i.e., biased estimates, and error covariance different from the true covariance [12]) (see [6]). To ensure correct observability properties of the proposed U-iSAM, we project the most accurate measurement Jacobians computed by the unscented transformation onto the observable subspace, while computing the propagation Jacobians in a standard way without projection. We validate the proposed U-iSAM in extensive Monte-Carlo simulations and show that it significantly out- 
performs the standard iSAM algorithm. It should be pointed out that apart from the CLATT problem considered in this paper, the proposed U-iSAM can be applicable for a large class of nonlinear estimation problems in robotics.

The remainder of the paper is organized as follows: In the next section, after mathematically formulating the CLATT problem, we explain the batch-MAP and the iSAM algorithms used to solve this problem. In Section III we perform the observability analysis for the batch-MAP-based CLATT system and analytically show the nullspace of the Hessian matrix. In Section IV we presents the proposed U-iSAM algorithm which introduces a key projection operation when computing the measurement Jacobians, and its performance is validated through Monte-Carlo simulations in Section V. Finally, Section VI outlines the main conclusions of this work, as well as possible future research directions.

\section{Problem Statement}

Consider a typical CLATT scenario of a team of robots moving in a plane and tracking multiple targets. Each of the tracking robots is equipped with both proprioceptive (e.g., an odometer) and exteroceptive (e.g., a laser scanner) sensors. In contrast, the targets do not have such onboard sensors, however, their motion is assumed to be a known stochastic model. The tracking robots measure distance and bearing measurements to the targets as well as the other robots in the team. Note that in this scenario we do not assume communication between robots, which, however, is not a necessary assumption and can be easily integrated to provide more measurement information. Using the available proprioceptive and exteroceptive measurements, we aim to estimate the state trajectory of both the robots and the targets.

The state vector of CLATT at time-step k contains all the robots' poses including positions and orientations, and the targets' kinematic states such as position and velocity, i.e.,

$$
\mathbf{x}(k)=\left[\begin{array}{lllllll}
\mathbf{x}_{R_{1}}^{T}(k) & \ldots & \mathbf{x}_{R_{N}}^{T}(k) & \mathbf{x}_{T_{1}}^{T}(k) & \ldots & \mathbf{x}_{T_{M}}^{T}(k)
\end{array}\right]^{T}
$$

where $\mathbf{x}_{R_{i}}^{T}:=\left[\begin{array}{ll}\mathbf{p}_{R_{i}}^{T} & \phi_{R_{i}}\end{array}\right]^{T}=\left[\begin{array}{lll}x_{R_{i}} & y_{R_{i}} & \phi_{R_{i}}\end{array}\right], i=1, \ldots, N$, denotes the $i$ th robot's pose (position and orientation); and $\mathbf{x}_{T_{i}}^{T}:=\left[\begin{array}{llll}\underbrace{x_{T_{i}}}_{\mathbf{p}_{T_{i}}^{T}} y_{T_{i}} & \underbrace{\dot{x}_{T_{i}}}_{\mathbf{d}_{T_{i}}^{T}} \dot{y}_{T_{i}} \ddot{x}_{T_{i}} \ddot{y}_{T_{i}} \cdots\end{array}\right], i=1, \ldots, M$, is the $i$ th target's kinematic states, including the target position, $\mathbf{p}_{T_{i}}$, and the higher-order time derivatives of the target position, $\mathbf{d}_{T_{i}}$. Hence, the state trajectory is given by stacking all the states from time-step 0 to $k$, i.e.,

$$
\mathbf{x}(0: k)=\left[\begin{array}{llll}
\mathbf{x}^{T}(0) & \mathbf{x}^{T}(1) & \cdots & \mathbf{x}^{T}(k)
\end{array}\right]^{T}
$$

\section{A. Robot motion model}

Each tracking robot is equipped with an odometer, whose measurement serves as the control input to propagate the robot pose based on the following motion model:

$$
\begin{aligned}
\mathbf{p}_{R_{i}}(k+1) & =\mathbf{p}_{R_{i}}(k)+\mathbf{C}\left(\phi_{R_{i}}(k)\right){ }^{R_{k}} \mathbf{p}_{R_{i, k+1}} \\
\phi_{R_{i}}(k+1) & =\phi_{R_{i}}(k)+{ }^{R_{k}} \phi_{R_{i, k+1}}
\end{aligned}
$$

where $\mathbf{C}(\cdot)$ denotes the $2 \times 2$ rotation matrix, and $\mathbf{u}_{i}(k)=$ ${ }^{R_{k}} \mathbf{x}_{R_{i, k+1}}=\left[{ }^{R_{k}} \mathbf{p}_{R_{i, k+1}}^{T}{ }^{R_{k}} \phi_{R_{i, k+1}}\right]^{T}$ is the true odometry (control input), i.e., the robot's motion between time-steps $k$ and $k+1$, expressed with respect to the robot's frame at timestep $k,\left\{R_{k}\right\}$. The corresponding odometry measurement, $\mathbf{u}_{m_{i}}(k)$, is commonly assumed to be corrupted by zeromean white Gaussian noise, $\mathbf{w}_{i}(k)=\mathbf{u}_{i}(k)-\mathbf{u}_{m_{i}}(k)$, with covariance $\mathbf{Q}_{R_{i}}(k)$. Clearly the above motion model is nonlinear and can be written in the following generic form:

$$
\mathbf{x}_{R_{i}}(k+1)=\mathbf{f}\left(\mathbf{x}_{R_{i}}(k), \mathbf{u}_{m_{i}}(k)+\mathbf{w}_{i}(k)\right)
$$

Linearization of the above function at the current state estimates yields the following Jacobians with respect to the entire state trajectory and noise, respectively: ${ }^{1}$

$$
\begin{aligned}
& \mathcal{F}_{R_{i}}(k)=\left[\begin{array}{llllll}
\mathbf{0} & \cdots & -\boldsymbol{\Phi}_{R_{i}}(k) & \mathbf{I}_{3} & \cdots & \mathbf{0}
\end{array}\right] \\
& \boldsymbol{\Phi}_{R_{i}}(k)=\left[\begin{array}{cc}
\mathbf{I}_{2} & \boldsymbol{\Gamma}\left(\hat{\mathbf{p}}_{R_{i}}(k+1 \mid k+1)-\hat{\mathbf{p}}_{R_{i}}(k \mid k)\right) \\
\mathbf{0}_{1 \times 2} & 1
\end{array}\right] \\
& \mathbf{G}_{R_{i}}(k)=\left[\begin{array}{cc}
\mathbf{C}\left(\hat{\phi}_{R_{i}}(k \mid k)\right) & \mathbf{0}_{2 \times 1} \\
\mathbf{0}_{1 \times 2} & 1
\end{array}\right]
\end{aligned}
$$

where $\Gamma:=\left[\begin{array}{cc}0 & -1 \\ 1 & 0\end{array}\right]$.

Note that this model (3) and (4) is general and encompasses all the motion models used in practice such as unicycle and Ackerman models. In particular, if the unicycle model is used, and we employ the approximation that the velocity and heading are constant during each propagation interval, we obtain ${ }^{R_{k}} \mathbf{x}_{R_{i, k+1}}=\left[\begin{array}{llll}v_{i}(k) \delta t & 0 & \omega_{i}(k) \delta t\end{array}\right]^{T}$, where $\mathbf{u}_{i}(k)=\left[\begin{array}{ll}v_{i}(k) & \omega_{i}(k)\end{array}\right]^{T}$ are the linear and rotational velocity, respectively, and $\delta t$ is the sampling period. Substitution in (3)-(4) yields the familiar propagation equations:

$$
\begin{aligned}
& \mathbf{p}_{R_{i}}(k+1)=\mathbf{p}_{R_{i}}(k)+\left[\begin{array}{l}
v_{i}(k) \delta t \cos \left(\phi_{R_{i}}(k)\right) \\
v_{i}(k) \delta t \sin \left(\phi_{R_{i}}(k)\right)
\end{array}\right] \\
& \phi_{R_{i}}(k+1)=\phi_{R_{i}}(k)+\omega_{i}(k) \delta t
\end{aligned}
$$

\section{B. Target motion model}

We consider the case where each of the targets moves randomly but assume that the stochastic model describing the motion of the target (e.g., constant acceleration or constant velocity) is known. In particular, the discrete-time state propagation equation is given by the following linear form:

$$
\mathbf{x}_{T_{i}}(k+1)=\boldsymbol{\Phi}_{T_{i}}(k) \mathbf{x}_{T_{i}}(k)+\mathbf{G}_{T_{i}}(k) \mathbf{w}_{T_{i}}(k)
$$

where $\mathbf{w}_{T_{i}}$ is zero-mean white Gaussian noise with covariance $\mathbf{Q}_{T_{i}}$. The state transition matrix, $\boldsymbol{\Phi}_{T_{i}}$, and the process noise Jacobian, $\mathbf{G}_{T_{i}}$, that appear in the preceding expression depend on the motion model used [12]. We will make no further assumptions on these matrices other than that their values are known. Similarly, the "Jacobian" matrix of (11) with respect to the state trajectory is given by:

$$
\mathcal{F}_{T_{i}}(k)=\left[\begin{array}{llllll}
\mathbf{0} & \cdots & -\boldsymbol{\Phi}_{T_{i}}(k) & \mathbf{I}_{\operatorname{dim}\left(\mathbf{x}_{T_{i}}\right)} & \cdots & \mathbf{0}
\end{array}\right]
$$

\section{Measurement model}

We consider the case where the tracking robots measure distance and bearing to the targets and the other robots in the team. The corresponding measurement equations are described in the following:

\footnotetext{
${ }^{1}$ Throughout this paper, the parenthesis $(\ell \mid j)$ refers to the estimate of a quantity at time-step $\ell$, after all measurements up to time-step $j$ have been processed. $\hat{x}$ denotes the estimate of a random variable $x$, while $\tilde{x}=x-\hat{x}$ is the error in this estimate. Finally, $\mathbf{0}_{m \times n}$ and $\mathbf{1}_{m \times n}$ denote $m \times n$ matrices of zeros and ones, respectively, and $\mathbf{I}_{n}$ is the $n \times n$ identity matrix.
} 
1) Robot-to-robot measurements: At time-step $k$ robot $i$ measures distance and bearing to robot $j$, which is given by:

$\mathbf{z}_{R_{i} R_{j}}(k)=\mathbf{v}_{i j}+$

$\left[\begin{array}{c}\sqrt{\left(x_{R_{j}}(k)-x_{R_{i}}(k)\right)^{2}+\left(y_{R_{j}}(k)-y_{R_{i}}(k)\right)^{2}} \\ \operatorname{atan} 2\left(\left(y_{R_{j}}(k)-y_{R_{i}}(k)\right),\left(x_{R_{j}}(k)-x_{R_{i}}(k)\right)\right)-\phi_{R_{i}}(k)\end{array}\right]$

$=\mathbf{h}\left(\mathbf{x}_{R_{i}}(k), \mathbf{x}_{R_{j}}(k)\right)+\mathbf{v}_{i j}$

where $\mathbf{v}_{i j}$ is zero-mean Gaussian measurement noise with covariance $\mathbf{R}_{R_{i j}}$. Linearization of (13) with respect to the state trajectory yields the following Jacobian matrix:

$$
\begin{aligned}
& \mathcal{H}_{R_{i j}}(k)=\left[\begin{array}{lllllll}
\mathbf{0} & \cdots & \mathbf{H}_{R_{i}}(k) & \cdots & \mathbf{H}_{R_{j}}(k) & \cdots & \mathbf{0}
\end{array}\right] \\
& \text { where } \quad \mathbf{H}_{R_{i}}(k)=\left[\begin{array}{cc}
\frac{\hat{\mathbf{p}}_{R_{i}}^{T}(k \mid k)-\hat{\mathbf{p}}_{R_{j}}^{T}(k \mid k)}{\left\|\hat{\mathbf{p}}_{R_{j}}^{T}(k \mid k)-\hat{\mathbf{p}}_{R_{i}}^{T}(k \mid k)\right\|} & \mathbf{0} \\
\frac{\left(\hat{\mathbf{p}}_{R_{i}}^{T}(k \mid k)-\hat{\mathbf{p}}_{R_{j}}^{T}(k \mid k)\right) \boldsymbol{\Gamma}^{T}}{\left\|\hat{\mathbf{p}}_{R_{j}}^{T}(k \mid k)-\hat{\mathbf{p}}_{R_{i}}^{T}(k \mid k)\right\|^{2}} & -1
\end{array}\right] \\
& \mathbf{H}_{R_{j}}(k)=\left[\begin{array}{cc}
\frac{\hat{\mathbf{p}}_{R_{j}}^{T}(k \mid k)-\hat{\mathbf{p}}_{R_{i}}^{T}(k \mid k)}{\left\|\hat{\mathbf{p}}_{R_{j}}^{T}(k \mid k)-\hat{\mathbf{p}}_{R_{i}}^{T}(k \mid k)\right\|} & 0 \\
\frac{\left(\hat{\mathbf{p}}_{R_{j}}^{T}(k \mid k)-\hat{\mathbf{p}}_{R_{i}}^{T}(k \mid k)\right) \boldsymbol{\Gamma}^{T}}{\left\|\hat{\mathbf{p}}_{R_{j}}^{T}(k \mid k)-\hat{\mathbf{p}}_{R_{i}}^{T}(k \mid k)\right\|^{2}} & \mathbf{0}
\end{array}\right]
\end{aligned}
$$

2) Robot-to-target measurements: Similarly, the robot-totarget distance and bearing measurement at time-step $k$ is:

$$
\begin{aligned}
& \mathbf{z}_{R_{i} T_{j}}(k)=\mathbf{n}_{i j}(k)+ \\
& {\left[\begin{array}{c}
\sqrt{\left(x_{T_{j}}(k)-x_{R_{i}}(k)\right)^{2}+\left(y_{T_{j}}(k)-y_{R_{i}}(k)\right)^{2}} \\
\operatorname{atan} 2\left(\left(y_{T_{j}}(k)-y_{R_{i}}(k)\right),\left(x_{T_{j}}(k)-x_{R_{i}}(k)\right)\right)-\phi_{R_{i}}(k)
\end{array}\right]} \\
& =: \mathbf{h}\left(\mathbf{x}_{R_{i}}(k), \mathbf{x}_{T_{j}}(k)\right)+\mathbf{n}_{i j}
\end{aligned}
$$

where $\mathbf{n}_{i j}$ is zero-mean white Gaussian measurement noise with covariance $\mathbf{R}_{T_{i j}}$. Analogously, the measurement Jacobian of (17), $\mathcal{H}_{T_{i j}}$, can be obtained by replacing $\hat{\mathbf{p}}_{R_{j}}$ in (14) by $\hat{\mathbf{p}}_{T_{j}}$, and has the following sparse structure:

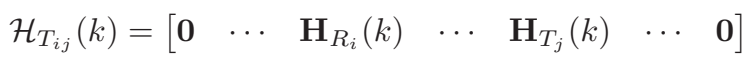

\section{Batch-MAP estimation}

In particular, we aim to estimate the entire state trajectory using all available information, which includes: (i) the prior information about the initial state, described by a Gaussian pdf with mean $\hat{\mathbf{x}}(0 \mid 0)$ and covariance $\mathbf{P}(0 \mid 0)$, (ii) the motion information [see (3), (4), and (11)], and (iii) the sensor measurements [see (13) and (17)]. To this end, the batchMAP estimator is often employed to determine the entire trajectory estimate $\hat{\mathbf{x}}(0: k \mid k)$ that maximizes the following posterior pdf:

$$
\begin{aligned}
& p(\mathbf{x}(0: k) \mid \mathbf{z}(0: k)) \propto p(\mathbf{x}(0)) \times \\
& \prod_{\kappa=0}^{k-1}\left[\prod_{i=1}^{N} p\left(\mathbf{x}_{R_{i}}(\kappa+1) \mid \mathbf{x}_{R_{i}}(\kappa)\right) \prod_{j=1}^{M} p\left(\mathbf{x}_{T_{j}}(\kappa+1) \mid \mathbf{x}_{T_{j}}(\kappa)\right)\right] \times \\
& \prod_{\kappa=0}^{k}\left[\prod_{\{i, j\} \in \mathcal{S}_{R}} p\left(\mathbf{z}_{R_{i} R_{j}}(\kappa) \mid \mathbf{x}_{R_{i}}(\kappa), \mathbf{x}_{R_{j}}(\kappa)\right) \times\right. \\
& \left.\prod_{\{i, j\} \in \mathcal{S}_{T}} p\left(\mathbf{z}_{R_{i} T_{j}}(\kappa) \mid \mathbf{x}_{R_{i}}(\kappa), \mathbf{x}_{T_{j}}(\kappa)\right)\right]
\end{aligned}
$$

where $\mathbf{z}(0: k)$ denotes all the sensor measurements available in the time interval $[0, k], \mathcal{S}_{R}:=\{i, j \mid i=1, \ldots, N ; j=$ $1, \ldots, N\}$, and $\mathcal{S}_{T}:=\{i, j \mid i=1, \ldots, N ; j=1, \ldots, M\}$. In the above expression (19), we have used the fact that state and measurement noise is independent and both the robot and target motion is a Markov process [see (5) and (11)]. Using the assumption of Gaussian noise, maximization of (19) is equivalent to minimizing the following cost function:

$$
\begin{aligned}
& c(\mathbf{x}(0: k)):=\frac{1}{2}\left\|\mathbf{x}_{0}-\hat{\mathbf{x}}_{0 \mid 0}\right\|_{\mathbf{P}_{0 \mid 0}}^{2}+ \\
& \frac{1}{2} \sum_{\kappa=0}^{k-1} \sum_{i=1}^{N}\left\|\mathbf{x}_{R_{i}}(\kappa+1)-\mathbf{f}\left(\mathbf{x}_{R_{i}}(\kappa), \mathbf{u}_{m_{i}}(\kappa)\right)\right\|_{\overline{\mathbf{Q}}_{R_{i}}(\kappa)}^{2}+ \\
& \frac{1}{2} \sum_{\kappa=0}^{k-1} \sum_{j=1}^{M}\left\|\mathbf{x}_{T_{j}}(\kappa+1)-\mathbf{\Phi}_{T_{j}} \mathbf{x}_{T_{j}}(\kappa)\right\|_{\overline{\mathbf{Q}}_{T_{j}}(\kappa)}^{2}+ \\
& \frac{1}{2} \sum_{\kappa=0}^{k} \sum_{\{i, j\} \in \mathcal{S}_{R}}\left\|\mathbf{z}_{R_{i} R_{j}}(\kappa)-\mathbf{h}\left(\mathbf{x}_{R_{i}}(\kappa), \mathbf{x}_{R_{j}}(\kappa)\right)\right\|_{\mathbf{R}_{R_{i j}}(\kappa)}^{2}+ \\
& \frac{1}{2} \sum_{\kappa=0}^{k} \sum_{\{i, j\} \in \mathcal{S}_{T}}\left\|\mathbf{z}_{R_{i} T_{j}}(\kappa)-\mathbf{h}\left(\mathbf{x}_{R_{i}}(\kappa), \mathbf{x}_{T_{j}}(\kappa)\right)\right\|_{\mathbf{R}_{T_{i j}}(\kappa)}^{2}
\end{aligned}
$$

where $\overline{\mathbf{Q}}_{R_{i}}:=\mathbf{G}_{R_{i}} \mathbf{Q}_{R_{i}} \mathbf{G}_{R_{i}}^{T}$ [see (5), (7), and (8)], and $\overline{\mathbf{Q}}_{T_{j}}:=\mathbf{G}_{T_{j}} \mathbf{Q}_{T_{j}} \mathbf{G}_{T_{j}}^{T}$ [see (11)]. In the above expressions, we have also employed the notation, $\|\mathbf{a}\|_{\boldsymbol{\Sigma}}^{2}:=\mathbf{a}^{T} \boldsymbol{\Sigma}^{-1} \mathbf{a}$, i.e., the squared Mahalanobis distance of residual a with covariance $\boldsymbol{\Sigma}$.

This is a nonlinear least-squares problem [see (5), (13), and (17)]. A standard iterative Newton-Raphson approach is often used for its optimization, although it is only able to converge to one local minimum within the basin of attraction of the initial estimate. Specifically, at the $\ell$-th iteration, a correction, $\delta \mathbf{x}^{(\ell)}(0: k)$, to the current estimate, $\hat{\mathbf{x}}^{(\ell)}(0: k \mid k)$, is computed by minimizing the second-order Taylor-series approximation of $(20):^{2}$

$$
\begin{aligned}
& c(\hat{\mathbf{x}}(0: k \mid k)+\delta \mathbf{x}(0: k)) \simeq \\
& c(\hat{\mathbf{x}}(0: k \mid k))+\mathbf{b}_{b} \delta \mathbf{x}(0: k)+\frac{1}{2} \delta \mathbf{x}^{T}(0: k) \mathbf{A}_{b} \delta \mathbf{x}(0: k)
\end{aligned}
$$

In the above equation, the Jacobian matrix of $c(\cdot)$ with respect to $\mathbf{x}(0: k), \mathbf{b}_{b}^{(\ell)}$, is computed and factorized as follows:

$$
\begin{aligned}
& \mathbf{b}_{b}=\mathbf{P}_{0 \mid 0}^{-1}(\hat{\mathbf{x}}(0 \mid k)-\hat{\mathbf{x}}(0 \mid 0))+ \\
& \sum_{\kappa=0}^{k-1} \sum_{i=1}^{N} \mathcal{F}_{R_{i}}^{T}(\kappa) \overline{\mathbf{Q}}_{R_{i}}^{-1}(\kappa)\left(\hat{\mathbf{x}}_{R_{i}}(\kappa+1 \mid k)-\mathbf{f}\left(\hat{\mathbf{x}}_{R_{i}}(\kappa \mid k), \mathbf{u}_{m_{i}}(\kappa)\right)\right)+ \\
& \sum_{\kappa=0}^{k-1} \sum_{i=1}^{M} \mathcal{F}_{T_{i}}^{T}(\kappa) \overline{\mathbf{Q}}_{T_{i}}^{-1}(\kappa)\left(\hat{\mathbf{x}}_{T_{i}}(\kappa+1 \mid k)-\mathbf{\Phi}_{T_{i}}(\kappa) \hat{\mathbf{x}}_{T_{i}}(\kappa \mid k)\right)+ \\
& \sum_{\kappa=0}^{k} \sum_{\{i, j\} \in \mathcal{S}_{R}} \mathcal{H}_{R_{i j}}^{(\ell)^{T}}(\kappa) \mathbf{R}_{R_{i j}}^{-1}\left(\mathbf{z}_{R_{i} R_{j}}(\kappa)-\mathbf{h}\left(\hat{\mathbf{x}}_{R_{i}}(\kappa \mid k), \hat{\mathbf{x}}_{R_{j}}(\kappa \mid k)\right)\right)+ \\
& \sum_{\kappa=0}^{k} \sum_{\{i, j\} \in \mathcal{S}_{T}} \mathcal{H}_{T_{i j}}^{(\ell)^{T}}(\kappa) \mathbf{R}_{T_{i j}}^{-1}\left(\mathbf{z}_{R_{i} T_{j}}(\kappa)-\mathbf{h}\left(\hat{\mathbf{x}}_{R_{i}}(\kappa \mid k), \hat{\mathbf{x}}_{T_{j}}(\kappa \mid k)\right)\right) \\
& =: \mathbf{J}^{T} \mathbf{r}
\end{aligned}
$$

\footnotetext{
${ }^{2}$ Note that hereafter we occasionally drop the time and iteration indices in order to make the presentation concise; these can be easily inferred from the context.
} 
where

$$
\begin{aligned}
& \mathbf{J}:=\left[\begin{array}{c}
\mathbf{P}_{0 \mid 0}^{-\frac{1}{2}} \\
\vdots \\
\overline{\mathbf{Q}}_{R_{i}}^{-\frac{1}{2}}(\kappa) \mathcal{F}_{R_{i}}(\kappa) \\
\vdots \\
\overline{\mathbf{Q}}_{T_{i}}^{-\frac{1}{2}}(\kappa) \mathcal{F}_{T_{i}}(\kappa) \\
\vdots \\
\mathbf{R}_{R_{i j}}^{-\frac{1}{2}}(\kappa) \mathcal{H}_{R_{i j}}(\kappa) \\
\vdots \\
\mathbf{R}_{T_{i j}}^{-\frac{1}{2}}(\kappa) \mathcal{H}_{T_{i j}}(\kappa) \\
\vdots
\end{array}\right]=\boldsymbol{\Lambda}\left[\begin{array}{c}
\mathbf{I} \\
\vdots \\
\mathcal{F}_{R_{i}}(\kappa) \\
\vdots \\
\mathcal{F}_{T_{i}}(\kappa) \\
\vdots \\
\mathcal{H}_{R_{i j}}(\kappa) \\
\vdots \\
\mathcal{H}_{T_{i j}}(\kappa) \\
\vdots
\end{array}\right] \\
& \mathbf{r}:=\left[\begin{array}{c}
\mathbf{P}_{0 \mid 0}^{-\frac{1}{2}}(\hat{\mathbf{x}}(0 \mid k)-\hat{\mathbf{x}}(0 \mid 0)) \\
\vdots \\
\overline{\mathbf{Q}}_{R_{i}}^{-\frac{1}{2}}(\kappa)\left(\hat{\mathbf{x}}_{R_{i}}(\kappa+1 \mid k)-\mathbf{f}\left(\hat{\mathbf{x}}_{R_{i}}(\kappa \mid k), \mathbf{u}_{m_{i}}(\kappa)\right)\right) \\
\vdots \\
\overline{\mathbf{Q}}_{T_{i}}^{-\frac{1}{2}}(\kappa)\left(\hat{\mathbf{x}}_{T_{i}}(\kappa+1 \mid k)-\boldsymbol{\Phi}_{T_{i}}(\kappa) \hat{\mathbf{x}}_{T_{i}}(\kappa \mid k)\right) \\
\vdots \\
\mathbf{R}_{R_{i j}}^{-\frac{1}{2}}(\kappa)\left(\mathbf{z}_{R_{i} R_{j}}(\kappa)-\mathbf{h}\left(\hat{\mathbf{x}}_{R_{i}}(\kappa \mid k), \hat{\mathbf{x}}_{R_{j}}(\kappa \mid k)\right)\right) \\
\vdots \\
\mathbf{R}_{T_{i j}}^{-\frac{1}{2}}(\kappa)\left(\mathbf{z}_{R_{i} T_{j}}(\kappa)-\mathbf{h}\left(\hat{\mathbf{x}}_{R_{i}}(\kappa \mid k), \hat{\mathbf{x}}_{T_{j}}(\kappa \mid k)\right)\right) \\
\vdots
\end{array}\right]
\end{aligned}
$$

In the above expression (23),

$\boldsymbol{\Lambda}:=\operatorname{Diag}\left(\mathbf{P}_{0 \mid 0}^{-\frac{1}{2}}, \cdots, \overline{\mathbf{Q}}_{R_{i}}^{-\frac{1}{2}}(\kappa), \cdots, \overline{\mathbf{Q}}_{T_{i}}^{-\frac{1}{2}}(\kappa), \cdots, \mathbf{R}_{R_{i j}}^{-\frac{1}{2}}(\kappa), \cdots, \mathbf{R}_{T_{i j}}^{-\frac{1}{2}}(\kappa), \cdots\right)$

for all $\kappa, i$, and $j$. On the other hand, during the commonlyused Gauss-Newton iterations, the Hessian matrix, $\mathbf{A}_{b}$, is approximated by:

$$
\begin{aligned}
& \mathbf{A}_{b}=\mathbf{P}_{0 \mid 0}^{-1}+ \\
& \sum_{\kappa=0}^{k-1} \sum_{i=1}^{N} \mathcal{F}_{R_{i}}^{T}(\kappa) \overline{\mathbf{Q}}_{R_{i}}^{-1}(\kappa) \mathcal{F}_{R_{i}}(\kappa)+\sum_{\kappa=0}^{k-1} \sum_{i=1}^{M} \mathcal{F}_{T_{i}}^{T}(\kappa) \overline{\mathbf{Q}}_{T_{i}}^{-1}(\kappa) \mathcal{F}_{T_{i}}(\kappa)+ \\
& \sum_{\kappa=0}^{k} \sum_{\{i, j\} \in \mathcal{S}_{R}} \mathcal{H}_{R_{i j}}^{T}(\kappa) \mathbf{R}_{R_{i j}}^{-1}(\kappa) \mathcal{H}_{R_{i j}}(\kappa)+\sum_{\kappa=0}^{k} \sum_{\{i, j\} \in \mathcal{S}_{T}} \mathcal{H}_{T_{i j}}^{T}(\kappa) \mathbf{R}_{T_{i j}}^{-1}(\kappa) \mathcal{H}_{T_{i j}}(\kappa) \\
& =: \mathbf{J}^{T} \mathbf{J}
\end{aligned}
$$

This is a good approximation for small-residual problems [13]. Due to the sparse structure of the matrices, $\mathcal{F}_{R_{i}}$, $\mathcal{F}_{T_{i}}, \mathcal{H}_{R_{i j}}$, and $\mathcal{H}_{T_{i j}}$ [see (6), (12), (14), and (18)], the matrix $\mathbf{A}_{b}$ is also sparse, which can be exploited to speed-up the solution of the linearized system.

In particular, based on (22) and (25), it is not difficult to show that minimization of (21) is equivalent to:

$$
\begin{gathered}
\min _{\delta \mathbf{x}(0: k)} c(\hat{\mathbf{x}}(0: k \mid k)+\delta \mathbf{x}(0: k)) \Leftrightarrow \\
\min _{\delta \mathbf{x}(0: k)}\|\mathbf{J} \delta \mathbf{x}(0: k)-\mathbf{r}\|^{2}
\end{gathered}
$$

We employ QR factorization to solve (26), i.e.,

$$
\begin{aligned}
& \min _{\delta \mathbf{x}(0: k)}\|\mathbf{J} \delta \mathbf{x}(0: k)-\mathbf{r}\|^{2}=\left\|\mathbf{Q}\left[\begin{array}{c}
\mathbf{R} \\
\mathbf{0}
\end{array}\right] \delta \mathbf{x}(0: k)-\mathbf{r}\right\|^{2} \\
& =\left\|\left[\begin{array}{c}
\mathbf{R} \\
\mathbf{0}
\end{array}\right] \delta \mathbf{x}(0: k)-\mathbf{Q}^{T} \mathbf{r}\right\|^{2}=:\left\|\left[\begin{array}{c}
\mathbf{R} \\
\mathbf{0}
\end{array}\right] \delta \mathbf{x}(0: k)-\left[\begin{array}{c}
\mathbf{d} \\
\mathbf{e}
\end{array}\right]\right\|^{2} \\
& \Leftrightarrow \min _{\delta \mathbf{x}(0: k)}\|\mathbf{R} \delta \mathbf{x}(0: k)-\mathbf{d}\|^{2}
\end{aligned}
$$

where we have used the reduced QR of J [14], i.e.,

$$
\mathbf{J}=\mathbf{Q}\left[\begin{array}{c}
\mathbf{R} \\
\mathbf{0}
\end{array}\right]=\left[\begin{array}{ll}
\mathbf{Q}_{1} & \mathbf{Q}_{2}
\end{array}\right]\left[\begin{array}{c}
\mathbf{R} \\
\mathbf{0}
\end{array}\right]=\mathbf{Q}_{1} \mathbf{R}
$$

Once $\delta \mathbf{x}^{(\ell)}(0: k)$ is found, the new estimate is updated as:

$$
\hat{\mathbf{x}}^{(\ell+1)}(0: k \mid k)=\hat{\mathbf{x}}^{(\ell)}(0: k \mid k)+\delta \mathbf{x}^{(\ell)}(0: k)
$$

Given an initial estimate $\hat{\mathbf{x}}^{(0)}(0: k \mid k)$ that resides within the attraction basin of the global optimum, this iterative algorithm computes the global minimum (i.e., MAP estimate) for the entire state trajectory.

\section{E. iSAM estimation}

As the robots continue moving and tracking, new measurements ${ }^{3}, \mathbf{z}_{R_{i} T_{j}}(k+1)$ (or $\mathbf{z}_{R_{i} R_{j}}(k+1)$ ), sequentially become available. In principle, we need to recompute the full Jacobian $\mathbf{J}$ and solve the batch MAP problem from scratch. However, this is an expensive operator, even by exploiting the sparsity of the Hessian matrix [5], [8], [15]. To save computations, we focus on the iSAM algorithm [7], which reuses the previously-computed Jacobian and incrementally updates the QR factorization directly.

In particular, we augment $\mathbf{J}$ (without recomputing it) with the new measurement Jacobian $\mathcal{H}_{T_{i j}}$ [see (28)]:

$$
\mathbf{J}_{a}:=\left[\begin{array}{c}
\mathbf{J} \\
\mathbf{R}_{T_{i j}}^{-\frac{1}{2}} \mathcal{H}_{T_{i j}}
\end{array}\right]=\left[\begin{array}{cc}
\mathbf{Q}_{1} & \mathbf{0} \\
\mathbf{0} & \mathbf{I}
\end{array}\right]\left[\begin{array}{c}
\mathbf{R} \\
\mathbf{R}_{T_{i j}}^{-\frac{1}{2}} \mathcal{H}_{T_{i j}}
\end{array}\right]
$$

We now aim to decompose $\mathbf{J}_{a}$ into triangular form (i.e., square-root information matrix). Since $\mathbf{J}$ was already factorized into the triangular $\mathbf{R}$, we only need to zero out the new block row, i.e., the new measurement Jacobian $\mathbf{H}_{T_{i j}}$, in order to obtain the updated square-root information matrix $\mathbf{R}_{a}$. This can be achieved efficiently, for example, by using Givens QR [14]. Similarly, the corresponding new vector, $\mathbf{d}_{a}$, can be obtained by applying the same Givens rotations to the augmented residual vector, $\mathbf{d}_{a}:=$ $\left[\begin{array}{c}\mathbf{d} \\ \mathbf{R}_{T_{i j}}^{-\frac{1}{2}}\left(\mathbf{z}_{R_{i} T_{j}}-\mathbf{h}\left(\hat{\mathbf{x}}_{R_{i}}, \hat{\mathbf{x}}_{T_{j}}\right)\right)\end{array}\right]$.

It is important to note that, although relinearization is not needed at each time step when a new measurement becomes available, in order to reduce the linearization errors, we relinearize the system at the latest, and thus the best, state estimates periodically [7] or as needed when the linearization point significantly deviates from the current state estimate [9]. In addition, we can combine variable reordering [7] with this batch factorization to reduce fillin of the resulting triangular system [see (27)], which can further speed up the subsequent incremental estimation.

\footnotetext{
${ }^{3}$ Note that the robot and target motion [see (5) and (11)] can be considered as a different type of measurements and hence can be treated analogously.
} 


\section{OBSERVABILITy ANALYSIS}

In this section, we examine the parameter observability properties [12] of the batch-MAP-based CLATT system, which, for the time being, is considered as a parameter (instead of state) estimation problem. The study of parameter observability examines whether the information provided by the available measurements is sufficient for estimating the parameters without ambiguity. When parameter observability holds, the Fisher information (Hessian) matrix is invertible. Since the information matrix describes the information available in the measurements, by studying its nullspace we can gain insights about the directions in the parameter (state) space along which information should be acquired.

In particular, the observability properties of the CLATT system are based on the following lemma:

Lemma 3.1: The Hessian matrix of the batch-MAP-based CLATT [see (25)] in the case of no prior at time-step $k$, has a nullspace of dimension three and is given by: ${ }^{4}$

$$
\mathbf{N}_{b}(k)=\operatorname{span}_{\text {col. }}\left[\begin{array}{cc}
\vdots & \vdots \\
\mathbf{I}_{2} & \boldsymbol{\Gamma} \hat{\mathbf{p}}_{R_{i}}(0 \mid k) \\
\mathbf{0} & 1 \\
\vdots & \vdots \\
\mathbf{I}_{2} & \boldsymbol{\Gamma} \hat{\mathbf{p}}_{R_{i}}(k \mid k) \\
\mathbf{0} & 1 \\
\mathbf{I}_{2} & \vdots \\
\mathbf{0} & \left(\mathbf{I}_{\operatorname{dim}\left(\mathbf{d}_{T_{i}}\right) / 2} \otimes \boldsymbol{\Gamma}\right) \hat{\mathbf{d}}_{T_{i}}(0 \mid k) \\
\vdots & \vdots \\
\mathbf{I}_{2} & \boldsymbol{\Gamma} \hat{\mathbf{p}}_{T_{i}}(k \mid k) \\
\mathbf{0} & \left(\mathbf{I}_{\operatorname{dim}\left(\mathbf{d}_{T_{i}}\right) / 2} \otimes \boldsymbol{\Gamma}\right) \hat{\mathbf{d}}_{T_{i}}(k \mid k) \\
\vdots & \vdots
\end{array}\right]
$$

where $\otimes$ denotes the Kronecker product.

Proof: We assume a variable ordering of first all the robot poses, then all the target states. This assumption is employed only to simplify the notation, and does not affect the results of the analysis, since the ordering of the variables does not change the rank of the Hessian matrix. Based on the structure of the Jacobians, $\mathcal{F}_{R_{i}}(6), \mathcal{H}_{R_{i j}}(14), \mathcal{H}_{T_{i j}}$ (18), it is easy to verify that $\mathcal{F}_{R_{i}} \mathbf{N}_{b}=\mathbf{0}, \mathcal{H}_{R_{i j}} \mathbf{N}_{b}=\mathbf{0}$, and $\mathcal{H}_{T_{i j}} \mathbf{N}_{b}=\mathbf{0}$; while in proving $\mathcal{F}_{T_{i}} \mathbf{N}_{b}=\mathbf{0}$ [see (12)], we use the fact that $\boldsymbol{\Phi}_{T_{i}}$ and $\left(\mathbf{I}_{\operatorname{dim}\left(\mathbf{x}_{T_{i}}\right) / 2} \otimes \boldsymbol{\Gamma}\right)$ are commutative (e.g., this can be seen from the constant-velocity motion model (35) which is the most commonly used in practice). These equalities immediately result in [see (23) and (25)]: $\mathbf{J N}_{b}=\mathbf{0} \Rightarrow \mathbf{0}=\mathbf{J}^{T} \mathbf{J} \mathbf{N}_{b}=\mathbf{A}_{b} \mathbf{N}_{b}$.

This result indicates that any small changes of the state along the directions spanned by the columns of $\mathbf{N}_{b}(k)$ cannot be discerned based on the available measurements. Similar to [6], the first two columns of $\mathbf{N}_{b}(k)$ describe global translations of the state, while the third one describes global

\footnotetext{
${ }^{4}$ Since we are interested in the information contained in the available measurements, we consider the case without prior (i.e., $\mathbf{P}_{0 \mid 0} \rightarrow \infty$ ). In this case, the first block row of the matrix $\mathbf{J}$ (23) corresponding to the prior becomes zeros and can be ignored without changing the rank of the matrix.
}

rotations and high-order kinematic states (e.g., velocity). We thus see that, without the use of prior information, the global pose and high-order kinematic states cannot be determined. On the other hand, when prior information is included (i.e., $\mathbf{P}(0 \mid 0)<\infty)$, the Hessian becomes full-rank [see (23) and (25)], and thus it is possible to uniquely determine estimates for all the state variables. These results agree with our intuition, that based on relative measurements alone, the state trajectory cannot be determined with respect to the global frame of reference. Therefore, when designing a new iSAM algorithm, we expect the estimator to have the similar observability properties as the batch-MAP estimator.

\section{UNSCENTED ISAM}

The unscented transformation is often used to reduce linearization errors for many nonlinear estimation problems [3]. Hence, we expect the similar gain when using it in the iSAM to compute the Jacobians, and thus the Hessian. On the other hand, we also expect that the resulting Hessian has similar observability properties as that of the batchMAP estimator, i.e., the nullspace has dimension three (see Section III). However, this generally is not the case. When numerically computing the dimension of the nullspace of the Hessian, we find that it is three only for first few time steps, and decreases quickly as more measurements become available. This indicates that the naive U-iSAM acquires nonexistent information from the available measurements, in the directions of the state space where no information is actually available, such as the global position and orientation, which degrades the performance.

To address the aforementioned issue, we explicitly enforce the desired observability properties on the unscented transformation. To this end, we first design a nullspace for the Hessian matrix of the U-iSAM, $\mathbf{N}(k)$, that has correct dimension three. Although ideally we would like to have such a nullspace to be the one for the batch-MAP estimator (31), this is not possible in the iSAM since it uses the smoothed state estimates (i.e., $\hat{\mathbf{x}}(\ell \mid k)$ where $\ell<k$, that is, using the information from future measurements). Instead, we choose the nullspace in the same form as that of the batch-MAP estimator (31) but computed using the propagated state estimates for all the times (i.e., $\hat{\mathbf{x}}(\ell+1 \mid \ell)$ ).

Once the desired nullspace, $\mathbf{N}(k)$, is determined, we are now to find the appropriate Jacobians. In particular, we compute the propagation Jacobians through the standard unscented transformation. By construction, these propagation Jacobians for both robots and targets, denoted by $\boldsymbol{\Phi}(k)$, will automatically satisfy the observability constraint, i.e., $\boldsymbol{\Phi}(k) \mathbf{N}(k)=\mathbf{0}$. In contrast, the measurement Jacobians (including the robot-to-robot measurement Jacobian and the robot-to-target measurement Jacobian), denoted by $\mathbf{H}(k)$, is designed to be the one closest to the true Jacobian while satisfying the observability constraint, i.e.,

$$
\begin{aligned}
\min _{\mathbf{H}(k)} & \left\|\mathbf{H}_{o}-\mathbf{H}(k)\right\|_{F}^{2} \\
\text { subject to } & \mathbf{H}(k) \mathbf{N}(k)=\mathbf{0}
\end{aligned}
$$


where $\|\cdot\|_{F}$ denotes the Frobenius norm, and $\mathbf{H}_{o}$ is the ideal measurement Jacobian evaluated at the true states. In practice, since the true states are unavailable, we instead compute it using the standard unscented transformation. It is important to point out that in order to save computations we should exploit the structure of the measurement Jacobians (14) and (18) and only consider the nonzero components of these Jacobians in formulating the problem (32)-(33). To this end, only the corresponding entries of the nullspace $\mathbf{N}(k)$ are used accordingly. The optimal, closed-form solution to the problem (32)-(33) is based on the following lemma:

Lemma 4.1: The optimal solution to the minimization problem (32) and (33) is given by:

$$
\mathbf{H}(k)=\mathbf{H}_{o}\left(\mathbf{I}_{\operatorname{dim}(\mathbf{x})}-\mathbf{N}\left(\mathbf{N}^{T} \mathbf{N}\right)^{-1} \mathbf{N}^{T}\right)
$$

Proof: See Appendix I.

It is interesting to note that, since $\mathbf{N}$ in (34) is the unobservable subspace (nullspace) at time-step $k$, $\left(\mathbf{I}_{\operatorname{dim}(\mathbf{x})}-\mathbf{N}\left(\mathbf{N}^{T} \mathbf{N}\right)^{-1} \mathbf{N}^{T}\right)$ is the subspace orthogonal to $\mathbf{N}$, i.e., the observable subspace at time-step $k$. Hence, as seen from (34), the measurement Jacobian, $\mathbf{H}(k)$, is the projection of the most accurate measurement Jacobian onto the observable subspace so that no spurious information is acquired from the measurements.

\section{Simulation Results}

A series of Monte-Carlo comparison studies were conducted under various conditions using different types of measurements in order to validate the proposed U-iSAM algorithm. In the simulation tests, we consider the CLATT scenario in which three robots randomly move in an area of size $60 \mathrm{~m} \times 60 \mathrm{~m}$ and track two targets. Fig. 1(a) shows an example of trajectories of the robots and the targets, which is obtained from one realization of the Monte-Carlo trials.

For the results presented in this section, three identical robots with a simple differential drive model start from the same place and move on a planar surface, at a constant linear velocity of $v=0.5 \mathrm{~m} / \mathrm{sec}$, while the rotational velocity is drawn from the uniform distribution over $[-0.5,0.5] \mathrm{rad} / \mathrm{sec}$. The standard deviation of the velocity measurement noise is equal to $\sigma_{v}=2 \% v$, while the rotational velocity measurements are corrupted by noise with standard deviation $\sigma_{\omega}=1$ $\mathrm{deg} / \mathrm{sec}$. On the other hand, we adopt a zero-acceleration target motion model [12]:

$$
\dot{\mathbf{x}}_{T_{i}}(t)=\mathbf{F}_{T_{i}} \mathbf{x}_{T_{i}}(t)+\mathbf{G}_{T_{i}} \mathbf{w}_{T_{i}}(t)
$$

where

$\mathbf{F}_{T_{i}}=\left[\begin{array}{cccc}0 & 0 & 1 & 0 \\ 0 & 0 & 0 & 1 \\ 0 & 0 & 0 & 0 \\ 0 & 0 & 0 & 0\end{array}\right], \mathbf{G}_{T_{i}}=\left[\begin{array}{cc}0 & 0 \\ 0 & 0 \\ 1 & 0 \\ 0 & 1\end{array}\right], \mathbf{x}_{T_{i}}(t)=\left[\begin{array}{c}x_{T_{i}}(t) \\ y_{T_{i}}(t) \\ \dot{x}_{T_{i}}(t) \\ \dot{y}_{T_{i}}(t)\end{array}\right]$

and $\mathbf{w}_{T_{i}}(t)=\left[\begin{array}{ll}w_{x}(t) & w_{y}(t)\end{array}\right]^{T}$ is zero-mean, white Gaussian noise with covariance $\mathbb{E}\left[\mathbf{w}_{T_{i}}(t) \mathbf{w}_{T_{i}}(\tau)^{T}\right]=q \mathbf{I}_{2} \delta(t-$ $\tau), q=0.01$, and $\delta(t-\tau)$ is the Dirac delta function. In our implementation, we discretize the continuous-time system model (35) with time step $\Delta t=1 \mathrm{sec}$. The initial true state of the target is $\mathbf{x}_{T_{i}}(0)=(-1)^{i}\left[\begin{array}{llll}10 & -10 & -0.1 & 0.1\end{array}\right]^{T}$,
TABLE I

Robot AND TARGET STATE Estimation ERRORS

\begin{tabular}{lccc}
\hline \hline & iSAM [7] & U-iSAM & MAP \\
\hline \hline \multicolumn{4}{c}{ Robot Position RMSE (m) } \\
\hline Robot 1: & 2.1218 & 1.7019 & 1.4820 \\
Robot 2: & 2.0390 & 1.7583 & 1.4757 \\
Robot 3: & 1.4663 & 1.0338 & 0.8127 \\
\hline \hline \multicolumn{4}{c}{ Robot Heading RMSE (rad) } \\
\hline Robot 1: & 0.1282 & 0.0892 & 0.0602 \\
Robot 2: & 0.1315 & 0.1055 & 0.0737 \\
Robot 3: & 0.1339 & 0.0907 & 0.0603 \\
\hline \hline & \multicolumn{4}{c}{ Target Position RMSE (m) } \\
\hline Target 1: & 2.8814 & 1.9731 & 0.8204 \\
Target 2: & 6.7421 & 3.8297 & 0.8245 \\
\hline \hline & \multicolumn{3}{c}{ Target Velocity RMSE (m/sec) } \\
\hline Target 1: & 0.2172 & 0.1357 & 0.0314 \\
Target 2: & 0.2922 & 0.1751 & 0.0293 \\
\hline \hline
\end{tabular}

$i=1,2$, while the initial estimate of the target state is set to $\hat{\mathbf{x}}_{T_{i}}(0 \mid 0) \sim \mathcal{N}\left(\mathbf{x}_{T_{i}}(0), \mathbf{P}_{T_{i}}(0 \mid 0)\right)$, with the initial covariance $\mathbf{P}_{T_{i}}(0 \mid 0)=\operatorname{Diag}\left(\mathbf{I}_{2}, 0.01 \mathbf{I}_{2}\right)$.

While any type of measurements (e.g., range-and-bearing, bearing-only, and range-only measurements) is applicable for the proposed algorithm, we here consider the case with significant nonlinearity, where each robot records distance measurements to all other robots and targets. Note that for simplicity we assume that each robot can observe all others at every time step. However, this is not a necessary assumption, as the analysis can easily be extended to the case where multiple propagation steps occur between updates (e.g., limited sensing range, or different sampling frequencies between proprioceptive and exteroceptive sensors). In this test, data association is also assumed to be known in order to focus on comparing the estimation performance. The standard deviation of the distance measurement noise is equal to $3 \%$ of the actual distance.

100 Monte-Carlo simulations were performed, and three estimators were compared: (i) the batch MAP, which is the best that can be achieved in practice and serves as the benchmark, (ii) the iSAM [7], and (iii) the proposed UiSAM. Note that both iSAMs relinearize and do a batch update every 20 time steps. The comparison results are shown in Fig. 1 and Table I. As expected, the batch MAP achieves the best accuracy in terms of root mean squared errors (RMSE). Most importantly, it becomes clear that the proposed U-iSAM performs better than the standard iSAM. This is attributed to the fact that the U-iSAM employs the unscented transformation (statistical linearization) to improve the accuracy of linearization approximation, while ensuring the correct observability properties so that no spurious information is gained from the available measurements (see Section IV). 


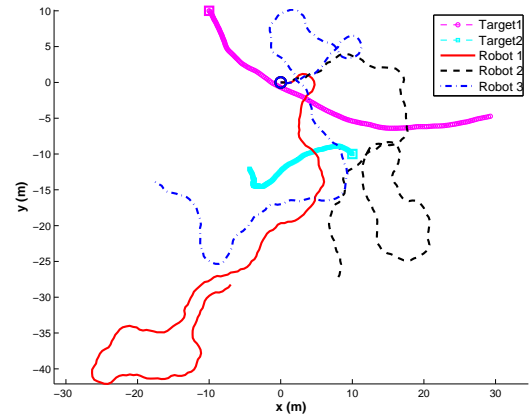

(a) Simulation setup
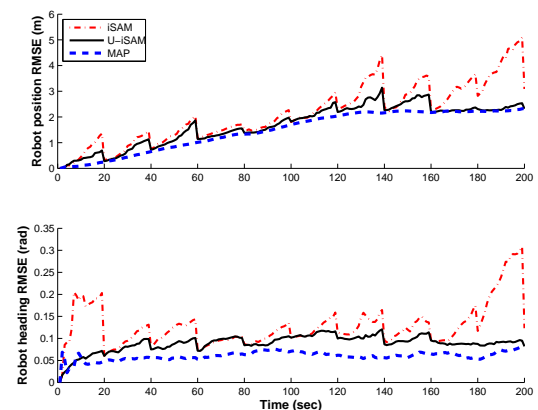

(b) Robot-1 RMSE
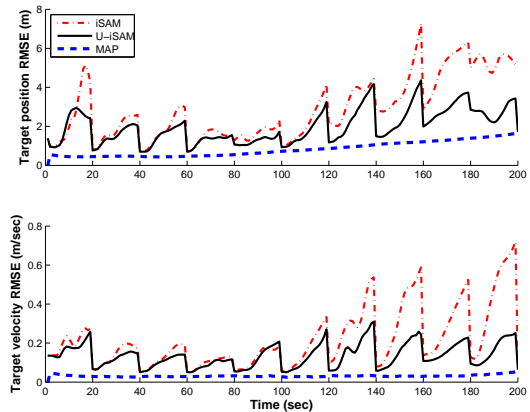

(c) Target-1 RMSE

Fig. 1. Monte-Carlo results for a CLATT scenario where three robots move randomly inside a $60 \mathrm{~m} \times 60 \mathrm{~m}$ arena and track two targets whose motion follows a zero-acceleration model. Starting positions of the robots are marked by $\bigcirc$ and those of the targets by $\square$. We show the estimation results only for one robot and one target, while the results for the other robots and target are similar to the ones presented here.

\section{CONCLUSiOnS AND Future Work}

In this paper, we have focused on the CLATT problem which is of practical significance when a team of robots operate in dynamic environments. In particular, we have analytically shown that the nullspace of the Hessian (information) matrix of the batch-MAP-based CLATT in the case of no prior, has dimension three. To ensure that the proposed U-iSAM, which employs the unscented transformation to compute the Jacobians and thus the Hessian, has the nullspace of the Hessian of correct dimensions, we project the most accurate measurement Jacobians computed by the standard unscented transformation onto the desired observable subspace. The proposed approach has been extensively tested in Monte-Carlo simulations and shown to perform significantly better than the standard iSAM. In the future, we plan to extend the analysis and algorithm to 3D.

\section{APPENDIX I}

\section{PROOF OF LEMMA 4.1}

The constraint (33) states that the rows of $\mathbf{H}$ lie in the left nullspace of the matrix $\mathbf{N}$. Therefore, if $\mathbf{L}$ is a matrix whose rows span this nullspace, then $\mathbf{H}$ can be written as:

$$
\mathbf{H}=\mathbf{\Theta} \mathbf{L}
$$

where $\Theta$ is the unknown matrix we seek to find. We note that there are several possible ways of computing an appropriate matrix $\mathbf{L}$, whose rows lie in the nullspace of $\mathbf{N}$. For instance, such a matrix is given, in closed form, by the expression:

$$
\mathbf{L}=\left[\begin{array}{ll}
\mathbf{I}_{m} & \mathbf{0}_{m \times(n-m)}
\end{array}\right]\left(\mathbf{I}_{n}-\mathbf{N}\left(\mathbf{N}^{T} \mathbf{N}\right)^{-1} \mathbf{N}^{T}\right)=: \mathbf{\Psi} \boldsymbol{\Pi}
$$

where $n$ is the dimension of the state and $m$ is the dimension of the measurement. It is not difficult to see that $\Pi:=$ $\mathbf{I}_{n}-\mathbf{N}\left(\mathbf{N}^{T} \mathbf{N}\right)^{-1} \mathbf{N}^{T}$ is an orthogonal projection matrix (i.e., $\Pi^{2}=\Pi$ and $\left.\Pi^{T}=\Pi\right)$ and hence has the eigenvalues of either 1 or 0 , whose reduced SVD is given by $\boldsymbol{\Pi}=\mathbf{U U}^{T}$. Using this result, $\mathbf{L}^{T}$ immediately can be written as $\mathbf{L}^{T}=$ $\mathbf{U U}^{T} \Psi^{T}$. By substituting this identity into the cost function, we have:

$$
\begin{aligned}
& \min \left\|\mathbf{H}_{o}-\mathbf{H}\right\|_{F}^{2}=\left\|\mathbf{U}^{T} \boldsymbol{\Psi}^{T} \boldsymbol{\Theta}^{T}-\mathbf{U}^{T} \mathbf{H}_{o}^{T}\right\|_{F}^{2} \\
& \Rightarrow \boldsymbol{\Theta}=\mathbf{H}_{o} \mathbf{U}(\mathbf{\Psi} \mathbf{U})^{-1}
\end{aligned}
$$

Therefore, substitution of the above equation in (36) yields (34). This completes the proof.

\section{REFERENCES}

[1] F. M. Mirzaei, A. I. Mourikis, and S. I. Roumeliotis, "On the performance of multi-robot target tracking," in Proc. of the IEEE International Conference on Robotics and Automation, Rome, Italy, Apr. $10-14,2007$, pp. 3482-3489.

[2] A. Sarwal, D. Agrawal, and S. Chaudhary, "Surveillance in an open environment by co-operative tracking amongst sensor enabled robots," in International Conference on Information Acquisition, Seogwipo-si, South Korea, Jul. 8-11, 2007, pp. 345-349.

[3] S. Julier, J. Uhlmann, and H. F. Durrant-Whyte, "A new method for the nonlinear transformation of means and covariances in filters and estimators," IEEE Transactions on Automatic Control, vol. 45, no. 3, pp. 477-482, Mar. 2000.

[4] T. Lefebvre, H. Bruyninckx, and J. De Schuller, "Comment on "a new method for the nonlinear transformation of means and covariances in filters and estimators" [and authors' reply]," IEEE Transactions on Automatic Control, vol. 47, no. 8, pp. 1406-1409, Aug. 2002.

[5] F. Dellaert and M. Kaess, "Square root SAM: Simultaneous localization and mapping via square root information smoothing," International Journal of Robotics Research, vol. 25, no. 12, pp. 1181-1203, Dec. 2006.

[6] G. P. Huang, A. I. Mourikis, and S. I. Roumeliotis, "An observability constrained sliding window filter for SLAM," in Proc. of the IEEE/RSJ International Conference on Intelligent Robots and Systems, San Francisco, CA, Sep. 25-30, 2011, pp. 65-72.

[7] M. Kaess, A. Ranganathan, and F. Dellaert, "iSAM: Incremental smoothing and mapping," IEEE Transactions on Robotics, vol. 24, no. 6, pp. 1365-1378, Dec. 2008.

[8] R. Kummerle, G. Grisetti, H. Strasdat, K. Konolige, and W. Burgard, "g20: A general framework for graph optimization," in Proc. of the IEEE International Conference on Robotics and Automation, Shanghai, China, May 9-13, 2011, pp. 3607-3613.

[9] M. Kaess, H. Johannsson, R. Roberts, V. Ila, J. Leonard, and F. Dellaert, "iSAM2: Incremental smoothing and mapping using the Bayes tree," International Journal of Robotics Research, vol. 31, pp. 217 236, Feb. 2012.

[10] H. Strasdat, J. M. M. Montiel, and A. J. Davison, "Visual SLAM: Why filter?" Image and Vision Computing, vol. 30, no. 2, pp. 65-77, Feb. 2012.

[11] S. Kay, Fundamentals of Statistical Signal Processing, Vol. I - Estimation Theory. Prentice Hall, 1993.

[12] Y. Bar-Shalom, X. R. Li, and T. Kirubarajan, Estimation with applications to tracking and navigation. New York: Wiley, 2001.

[13] B. Triggs, P. F. McLauchlan, R. I. Hartley, and A. W. Fitzgibbon, "Bundle adjustment - a modern synthesis," Lecture Notes in Computer Science, vol. 1883, pp. 298-375, Jan. 2000.

[14] G. H. Golub and C. F. V. Loan, Matrix Computations. The Johns Hopkins University Press, 1996.

[15] K. Konolige, G. Grisetti, R. Kummerle, W. Burgard, B. Limketkai, and R. Vincent, "Efficient sparse pose adjustment for 2D mapping," in Proc. of the IEEE/RSJ International Conference on Intelligent Robots and Systems, Taipei, Taiwan, Oct. 18-22, 2010, pp. 22-29. 